\title{
InterViewer: Dynamic Visualization of Protein-Protein Interactions ${ }^{\star}$
}

\author{
Kyungsook Han ${ }^{1}$, Byong-Hyon Ju${ }^{1}$, and Jong H. Park ${ }^{2}$ \\ 1 Department of Computer Science \& Engineering, Inca University \\ Inchon 402-751, South Korea \\ 2 MRC-DUNN, Human Nutrition Unit, Cambridge CB2 2XY, UK \\ http://wilab.inha.ac.kr/protein/
}

\section{Short Description}

From the perspective of graph drawing, a protein interaction network is challenging because it is a complicated, nonplanar graph with a large number of edge crossings and because it is a disconnected graph consisting of many connected components with nodes of a wide range of degrees. INTER VIEWER is a system for drawing and exploring protein-protein interaction networks in three-dimensional space. Unique features of INTERVIEWER include: (1) it is much faster than other recent implementations of drawing algorithms; (2) it can be used not only for drawing protein interaction networks but also for simplifying them into different types of networks; and (3) it provides an integrated framework for querying protein interaction databases and directly visualizing the query results.

\section{Areas of Application}

INTERVIEWER has been developed to visualize and analyze protein-protein interactions. Uses of INTERVIEWER include:

- visualizing large protein interaction networks and exploring them in 3D by rotating or by zooming in or out of them,

- finding proteins sharing a function or proteins interacting with a target protein within a specified distance level,

- comparing two or more protein interaction networks,

- creating a local database of protein-protein interactions, and

- producing protein interaction networks for publications or presentations

\section{Layout Algorithms and Layout Features}

INTERVIEWER's layout is based on the force-directed layout of Walshaw's algorithm [2], but different from Walshaw's algorithm in the following sense: (1)

\footnotetext{
* This work was supported by the advanced backbone IT technology development program of the Ministry of Information and Communication of Korea under grant number IMT2000-C3-4.
} 


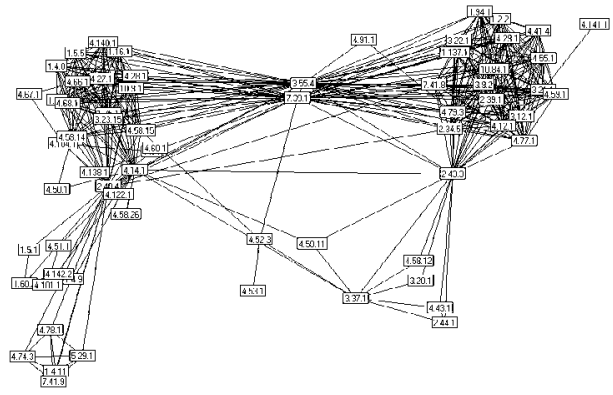

(A)

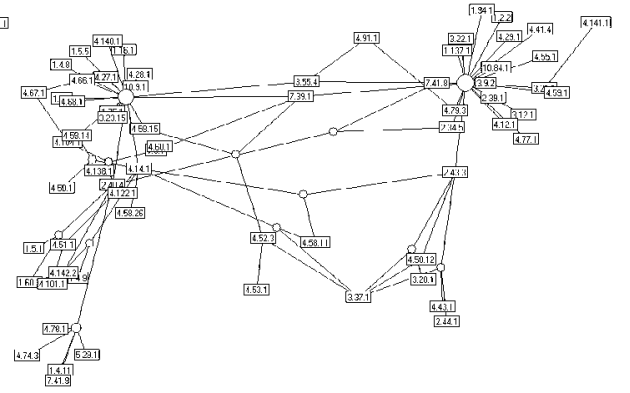

(B)

Fig. 1. (A) Subgraph of PSIMAP [1] obtained by extracting protein families interacting with protein family 7.39.1 within distance level of 2. (B) Simplified graph of Fig. TA by replacing a complete subgraph with a star-shaped subgraph centered at a dummy node, shown as a circle.

Walshaw's algorithm groups nodes into clusters, whereas InterViewer does not. (2) Walshaw's algorithm initially places nodes randomly, whereas InterViewer places nodes on the surface of a sphere for better results. (3) Walshaw's algorithm iteratively updates layouts until the graph size falls below a certain threshold value, whereas InterViewer iterates 20 times unless specified otherwise by a user.

Subgraphs with nodes of high degree often reduce the readability of the graph due to cluttered edges. We simplify this type of graph by replacing a complete subgraph with a star-shaped subgraph. While a complete graph with $n$ nodes contains $n(n-1) / 2$ edges, a star-shaped graph with $n$ nodes contains exactly $n$ edges. Thus, replacing complete subgraphs with star-shaped subgraphs substantially reduces the number of edges (see Fig. 1).

\section{Architecture and Interfaces}

The layout algorithm was implemented in Borland Delphi 6.0, and databases of protein-protein interactions were constructed using Microsoft Data Access Components 2.7. INTERVIEWER runs on Windows 2000/XP/Me/98/NT 4.0. INTERVIEWER is not coupled with a specific database. The user can easily make his own interaction databases using our program called DATASERVER and exploit them with INTER Viewer. DATASERVER and INTERVIEWER can run on a same computer or on different computers. Details are at http://wilab.inha.ac.kr/protein/.

\section{References}

1. Park, J., Lappe, M., Teichmann, S.A.: Mapping protein family interactions: intramolecular and intermolecular protein family interaction repertoires in the PDB and Yeast. J. Mol. Biol. 307 (2001) 929-938

2. Walshaw, C.: A multilevel Algorithm for Force-Directed Graph Drawing. In Proc. 8th Int. Symp. Graph Drawing, LNCS 1984, (2002) 171-182 